\title{
Relation of cognitive reserve and task performance to expression of regional covariance networks in an event-related fMRI study of nonverbal memory is
}

\author{
Christian Habeck, ${ }^{\mathrm{a}}$ H. John Hilton, ${ }^{\mathrm{a}}$ Eric Zarahn, ${ }^{\mathrm{a}, \mathrm{c}}$ Joseph Flynn, ${ }^{\mathrm{a}}$ \\ James Moeller, ${ }^{\mathrm{a}, \mathrm{c}}$ and Yaakov Stern ${ }^{\mathrm{a}, \mathrm{b}, \mathrm{c}, *}$ \\ ${ }^{a}$ Cognitive Neuroscience Division of the Taub Institute for Research in Alzheimer's Disease and the Aging Brain, \\ College of Physicians and Surgeons of Columbia University, New York, NY 10032, USA \\ ${ }^{\mathrm{b}}$ Department of Neurology, College of Physicians and Surgeons of Columbia University, New York, NY 10032, USA \\ ${ }^{c}$ Department of Psychiatry, College of Physicians and Surgeons of Columbia University, New York, NY 10032, USA
}

Received 23 January 2003; revised 28 July 2003; accepted 28 July 2003

\begin{abstract}
Cognitive reserve (CR) has been established as a mechanism that can explain individual differences in the clinical manifestation of neural changes associated with aging or neurodegenerative diseases. CR may represent individual differences in how tasks are processed (i.e., differences in the component processes), or in the underlying neural circuitry (of the component processes). CR may be a function of innate differences or differential life experiences. To investigate to what extent CR can account for individual differences in brain activation and task performance, we used fMRI to image healthy young individuals while performing a nonverbal memory task. We used IQ estimates as a proxy for CR. During both study and test phase of the task, we identified regional covariance patterns whose change in subject expression across two task conditions correlated with performance and CR. Common brain regions in both activation patterns were suggestive of a brain network previously found to underlie overt and covert shifts of spatial attention. After partialing out the influence of task performance variables, this network still showed an association with the CR, i.e., there were reserve-related physiological differences that presumably would persist were there no subject differences in task performance. This suggests that this network may represent a neural correlate of CR. (C) 2003 Elsevier Inc. All rights reserved.
\end{abstract}

\section{Introduction}

Several studies have suggested that differential susceptibility to age-related memory changes and dementia is related to variables such as education and IQ (Elias et al., 2000; Qiu et al., 2001; Scarmeas et al., 2001; Schmand et al., 1997). These studies provide epidemiological evidence for the presence of cognitive reserve (CR), where subjects with greater reserve may show less severe effects of the aging process. Cognitive reserve may represent individual differences in how tasks are processed (i.e., differences in the component processes), or in the underlying neural cir-

\footnotetext{
is Supported by NIA grant AG 14671 .

* Corresponding author. 630 West 168th Street, P\&S Box 16, New York, NY, 10032. Fax: +1-212-305-2426.

E-mail address: ysll@columbia.edu (Y. Stern).
}

cuitry (of the component processes). These differences may result from innate differences in intelligence or they may be acquired through life events such as educational or occupational experience. Different levels of reserve would result in some subjects being less susceptible to the effects of aging or pathology than others (Stern, 2002; Stern et al., 2003).

In this scheme, CR would be equally present in similarly aged individuals who are unaffected by any disease pathology. In contrast to the epidemiologic literature, recent cognitive neuroimaging research dealing with the aging process (Cabeza et al., 1997a,b; Reuter-Lorenz, 2002; Stern et al., 2000) highlighted many age-related differences in brain activation, but did not explicitly relate these findings to CR. To our knowledge there is only one study so far (Scarmeas et al., 2003) that could associate age-related differences between young and elderly with $\mathrm{CR}$.

Prompted by the finding of a relation between CR and 
age-related activation differences between age groups, we asked whether CR might also affect differences in activation within one age group and potentially account for variability that is routinely observed during performance of cognitive neuroimaging tasks. Focusing on one age group should keep the cumulative $\mathrm{CR}$ aspect of life experience constant, while allowing an investigation of the innate component of $\mathrm{CR}$ that is already present at low age. In particular, young subjects would be expected to show differences in neural activation during task processing that might be related to differences in CR measures such as IQ or education. Rather than focusing on differences in functional connectivity across groups (Cabeza et al., 1997a, 2002; Grady et al., 1999), we set out to map out commonalties in functional connectivity within a group of health young adults. We predicted that the level of expression of the same activation pattern would be related to the level of CR in each subject.

We used fMRI to examine healthy young adults during performance of a nonverbal serial recognition test. There were two task conditions. The low demand (L) condition required encoding and recognition of single items. The titrated demand $(\mathrm{T})$ condition required the subject to encode a larger list of items. Prior to scanning, this study list size (SLS) was adjusted for each subject, such that recognition accuracy was $75 \%$. This procedure was intended to control for task difficulty, matching difficulty across subjects. Our intention was to explore how individual differences in measured CR are related to changes in expression of a reserveand task-related brain network from the low to the titrated demand task.

We have previously reported univariate General Linear Model (GLM) analyses (Stern et al., 2003) that demonstrated a correlation between IQ and task-related activation differences between low and titrated demand conditions. In contrast, the regional covariance analysis employed in this paper first aimed to identify a network that underlies task performance in the experiment. We then determined whether individual expression of this network differed as a function of our reserve variables. Therefore, our method offers a more parsimonious account than the different voxels identified on the sole basis of the correlation of their activation with $\mathrm{CR}$ variables in the univariate analysis. Specifically, a task-related network whose expression also correlates with $\mathrm{CR}$ would provide a more convincing demonstration of having isolated a true neural substrate of CR via manipulation through an experimental design. The type-I error of erroneously detecting such a confluence of factors should be smaller than in univariate analyses where voxels of CR-related activation might bear no relationship to the task performed in the cognitive experiment.

Because univariate and multivariate analyses are truly complementary and focus on different signatures of the BOLD signal, it is difficult to judge whether they give congruent or incongruent results on the basis of statistically significant voxels identified in both analyses. For a scenario of very good experimental control it might be conceivable that a covariance pattern can be isolated whose highest voxel weights also exhibit significant group differences in a univariate analysis. However, the converse seems to be true more often: when several cognitive processes are simultaneously operational, only one of which is successfully manipulated by the experimental design, univariate and multivariate analyses might identify different brain regions. This is understandable given their different premises.

\section{Methods}

Seventeen healthy young adults between the ages of 21 and 30 participated. All subjects were carefully screened to ensure that they had no neurological or psychiatric disease. All subjects were right-handed. Informed consent was obtained after the nature and risks of the study were explained.

\section{Reserve indices}

Three variables were used as proxy measures for each subject's level of CR: years of education, and two indices of intelligence, the score on the New Adult Reading Test (NART) - North American version, and age-scaled score on the vocabulary subtest of the Wechsler Adult Intelligence Scale-Revised (WAIS-R). The two later tests are considered to be good estimates of verbal IQ.

\section{Activation task conditions}

Two conditions of a continuous performance nonverbal recognition test were considered in the current analysis. The basic task consisted of the serial presentation of one or more single unnamable shapes, followed by a series of the same number of recognition probes. The probes were distinguished from study items by a circumscribed rectangular frame. Study shapes were displayed for a duration of $4 \mathrm{~s}$. A $500 \mathrm{~ms}$ delay occurred at the transition to test trials, after which the recognition probes were displayed. A subject was instructed to make a "new" or "old" response for each probe item by pressing one of two buttons with the right or left finger. A LUMItouch optical device that was appropriate for use in a MRI setting was used for this purpose. A 6-s response time limit was imposed, with a premature response cutoff time of $200 \mathrm{~ms}$. Accuracy was stressed over speed. A new test probe was displayed at the end of the response time limit. New and old test probes occurred with the frequency of $50 \%$. Test probes were pseudorandomized so that no more than four consecutive trials required the same response.

Each shape was used only once for each subject. Shapes were designed to have similar characteristics that varied randomly within given sets of parameters. Loop shapes were used because their level of complexity made verbal encoding difficult (Fig. 1). The stimuli were prescreened to ensure that they could not easily be given a name. 

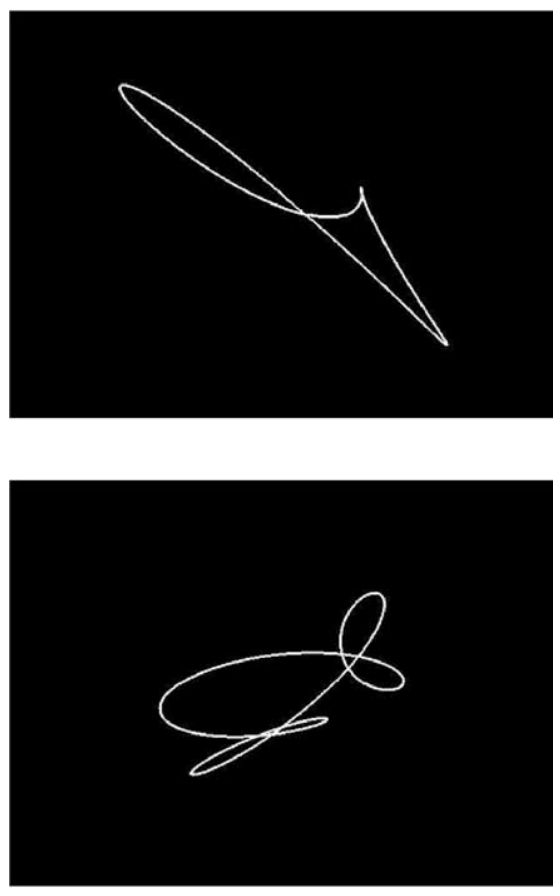

Fig. 1. Examples of loop shapes used as stimuli in the nonverbal memory task.

In the low demand condition, the study list size was 1 (i.e., one study shape followed by one recognition probe). The titrated demand condition used an SLS that was prede-
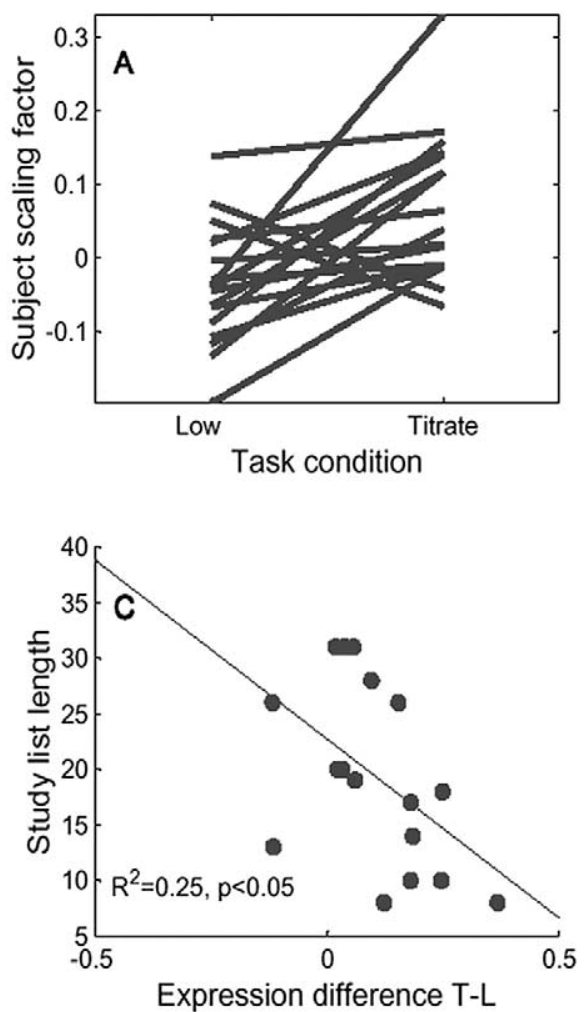

termined to elicit a recognition accuracy of $75 \%$ for the subject being tested. Prior to the fMRI scan, each subject completed a standardized training session and then two 15-min titration sessions. During these sessions, the particular SLS value at which the subject achieved $75 \%$ accuracy was determined using a staircase method of SLS adjustment. This SLS value was then used in the titrated demand condition on the day of the scan.

\section{fMRI scan acquisition}

Three 8-min task blocks were run. Within each 8-min block, 4 min were devoted to the titrated demand condition allowing 60 stimulus presentations (events) in total for both study and test. The number of events for the individual phases of the $\mathrm{T}$ condition therefore varies according to the achieved list length at which the subject reaches an accuracy of $75 \%$; the net number of events in the $\mathrm{T}$ condition can be different for study and test phase. There were 60 events available for the $T$ condition; if, say a subject has a study list length of 15 , this gives room for two study and two test sessions of 15 epochs each. If the subject has a list length of 16 , this gives room for two study sessions of 16 events each, as well as one test session of 16 events and a truncated test session of 12 events. A separate task that is unrelated to the current report was always interpolated between $\mathrm{T}$ and $\mathrm{L}$ task. This task took $2 \mathrm{~min}$. The remaining 2 min were devoted to the low demand task, which
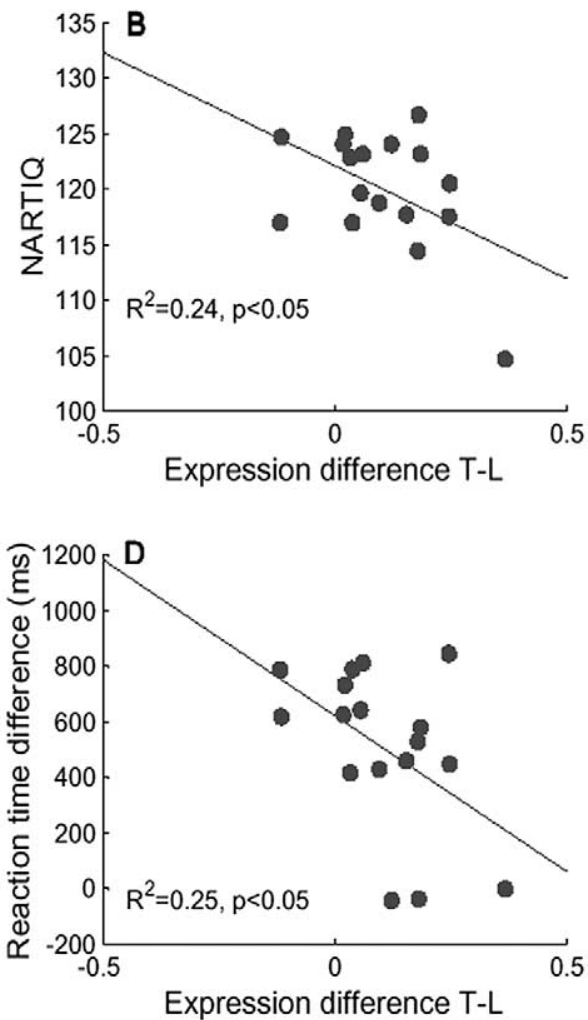

Fig. 2. Study phase: expression of covariance pattern found during study. (A) Increase in subject expression from $\mathrm{L}$ to $\mathrm{T}$ condition for 15 of 17 subjects; negative correlation of this increase in expression with (B) NARTIQ, (C) SLS, and (D) reaction time increase from L to T. 

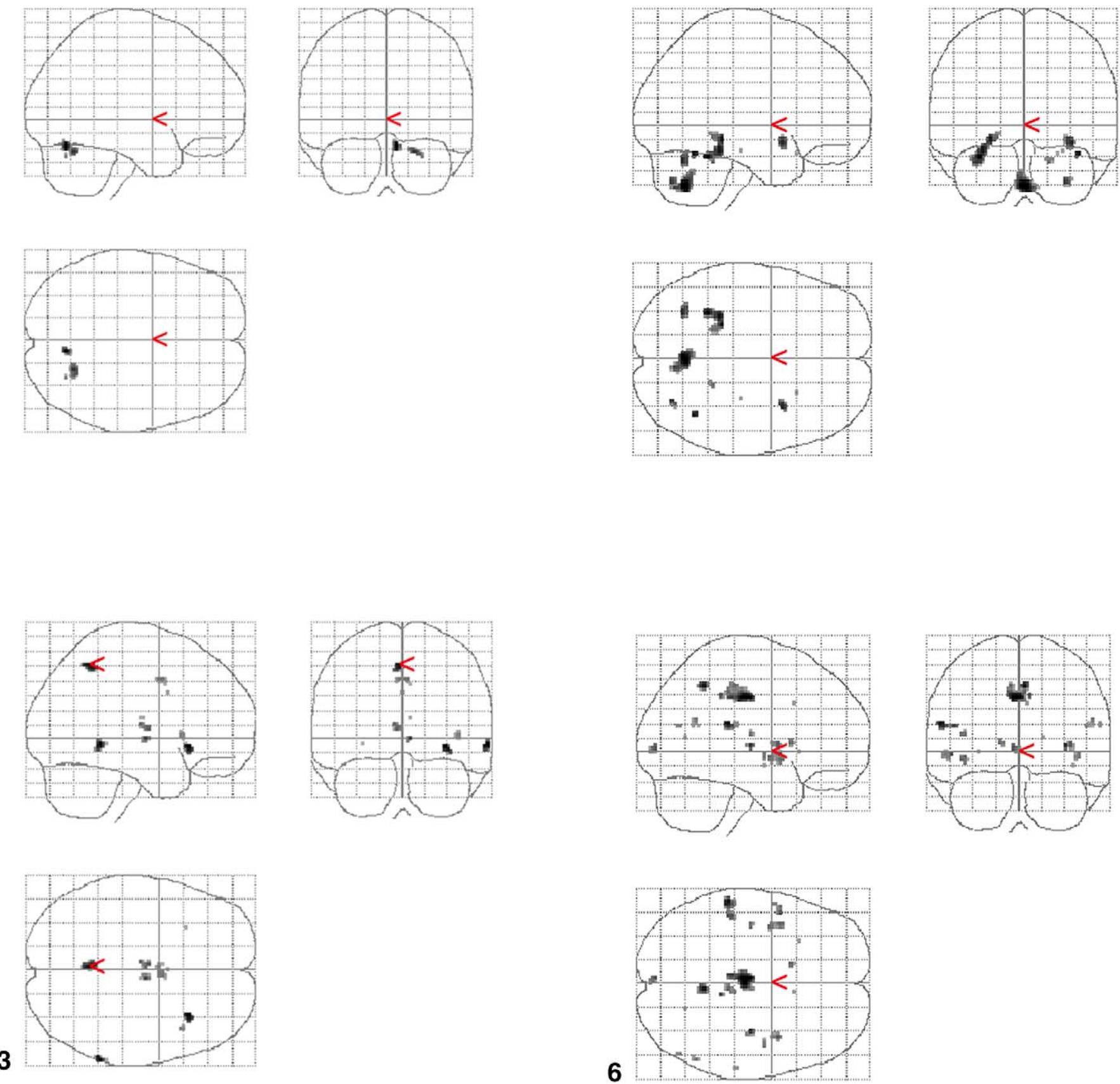

Fig. 3. Glass-brain projection views of positive and negative regional weights whose contribution to the covariance pattern is significant at $P<0.05$ as ascertained by a bootstrap test. The anatomical loci are listed in Table 1. Positive weights are shown in the upper part, negative weights in the lower part of the figure.

Fig. 6. Positive and negative regional weights whose contribution to the second principal component is significant at $P<0.05$ as ascertained by a bootstrap test. The anatomical loci are listed in Table 2. Positive weights are shown in the upper part, negative weights in the lower part of the figure. The positive weights of this topography contain parts of the positive weights of the study phase network and vice versa.

allowed seven events in the study and the test phase, respectively. A series of "blanks" (presentations of a bare cross hair) were randomly inserted into the study and test sequence for fMRI analytic purposes.

\section{MRI data acquisition}

Functional images were acquired using the 1.5 Tesla magnetic resonance scanner (General Electric) retrofitted for echoplanar imaging. A gradient echo EPI sequence (TE $=50 \mathrm{~ms} ; \mathrm{TR}=3 \mathrm{~s}$; flip angle $=90^{\circ}$ ) and a standard quadrature head coil was used to acquire $\mathrm{T} 2 *$ weighted images with an in-plane resolution of $3.124 \mathrm{~mm} \times 3.124$ $\mathrm{mm}\left(64 \times 64\right.$ matrix; $20 \mathrm{~cm}^{2}$ field of view). Based on $\mathrm{T}_{1}$ "scout" images, 15 to $178-\mathrm{mm}$ transaxial slices were acquired. Following the fMRI runs, a high resolution $\mathrm{T}_{2}$ image at the same slice locations used in the fMRI run was ac- 


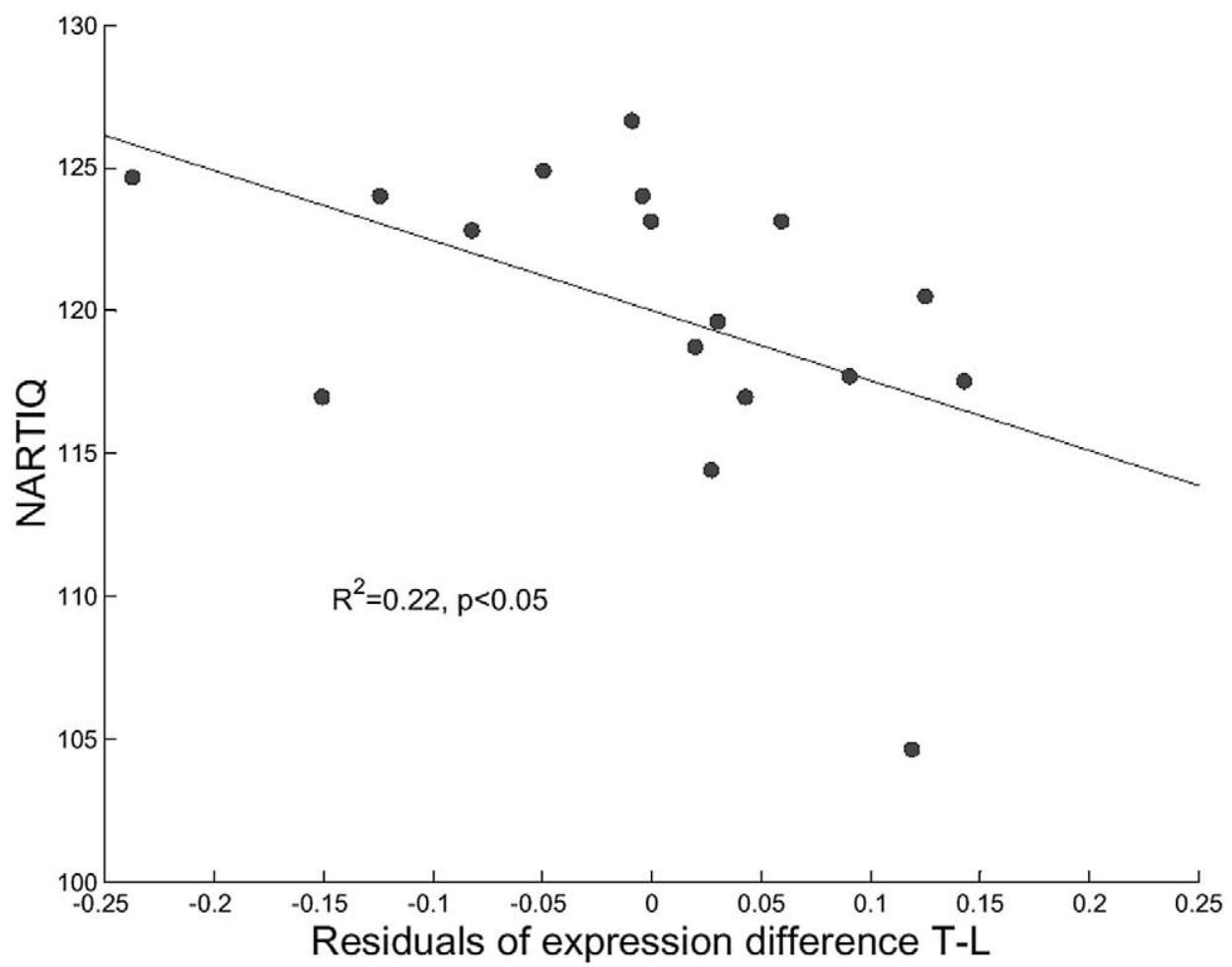

Fig. 4. Correlation between NARTIQ and the residuals of the study topography's expression after removal of the influence of SLS and reaction difference $\mathrm{T}$-L. The correlation is still significant, hinting at a pure association between $\mathrm{CR}$ and brain activation from $\mathrm{L}$ to $\mathrm{T}$.

quired using a fast spin echo sequence $[\mathrm{TE}=105 \mathrm{~ms}$; TR $=2500 \mathrm{~ms} ; 256 \times 256$ matrix; $20 \mathrm{~cm}^{2}$ field of view].

\section{fMRI processing}

All image processing and analysis were done using the statistical parametric mapping program (SPM 99, Wellcome Department of Cognitive Neurology) and other code written in Matlab 5.3 (Mathworks, Natick, MA). FMRI data were corrected for order of slice acquisition. All functional volumes in a given subject were realigned to the first volume from the first run of each study and SINC interpolation was used to reslice the images. The $\mathrm{T}_{2}$ high-resolution anatomical image was then coregistered to the aligned mean functional volume, using the mutual information coregistration algorithm implemented in SPM 99. This coregistered high resolution image was then used to determine nonlinear spatial normalization $(7 \times 8 \times 7$ nonlinear basis functions $)$ parameters for a transformation into a Talairach standard space defined by the Montreal Neurological Institute template brains applied with SPM 99. These normalization parameters were then applied to the functional data.

Contrasts assess the amplitudes (at every voxel in the image) of the components of the event-related responses that matched a canonical hemodynamic response waveform (a sum of two gamma functions, as specified in the SPM99 program). That is, the contrast weights for the Fourier predictor variables were chosen such that the resulting linear combination of the parameter estimates was an estimate of the desired linear combination of canonical hemodynamic response amplitudes (Zarahn, 2000, 2002). Modeling the time course of the hemodynamic response and computing its overlap with each voxel's activation time series in this way reduces the number of images to one per subject per condition, and thus makes a multivariate analysis feasible (which otherwise would be computationally impractical) at the population level. The within-subject contrast images entered into the multivariate analysis were the following: L vs blank trials and T vs blank trials. Study and test phases were analyzed separately.

\section{Regional covariance analysis}

Ordinal Trend Canonical Variates Analysis (OrT CVA) (Habeck et al., 2002) was performed on the data. This analysis is methodologically similar to other current regional covariance analyses techniques (Alexander et al., 1999; McIntosh et al., 1996; Worsley et al., 1997) and was designed to identify a covariance pattern that shows an ordinal trend, i.e., whose expression increases from the $\mathrm{L}$ to $\mathrm{T}$ condition for as many subjects as possible To achieve this, selected principal components from the covariance decomposition of subjects' $\mathrm{L}+\mathrm{T}$ data serve as predictors in a discriminant analysis that constructs a linear combination network whose expression differs maximally on the mean between the $\mathrm{L}$ and $\mathrm{T}$ condition. Which specific principal 

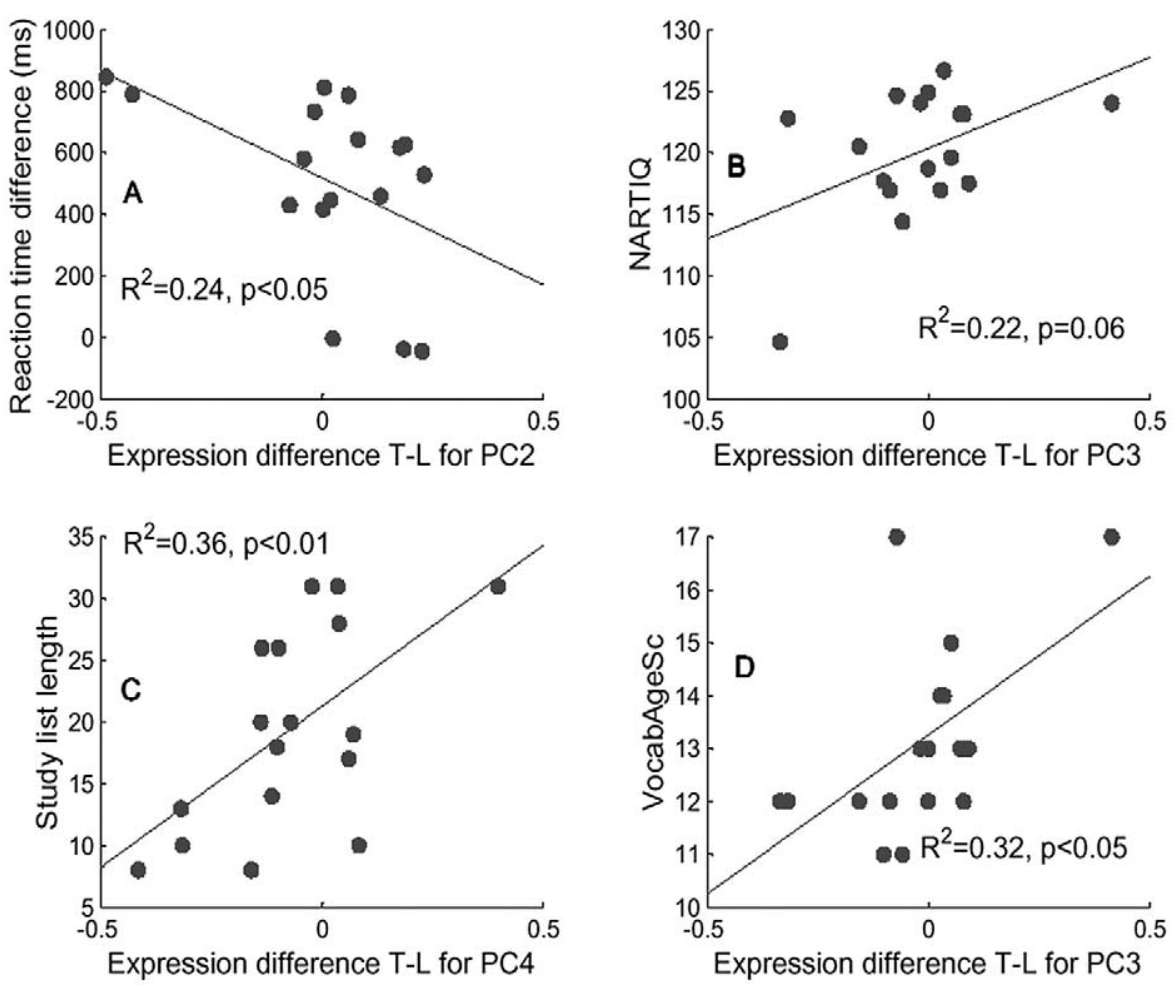

Fig. 5. Test phase: expression of principal components 2, 3, and 4 obtained, and the correlation of their increase in expression from L to T condition with reaction time increase, CR variables, and SLS, respectively.

components, $\mathrm{PC}_{1}-\mathrm{PC}_{K}, K=1 \ldots 6$, are included in the discriminant analysis, was decided on the basis of the Akaike information criterion (AIC) for best model selection (Burnham and Anderson, 2002). Consistent increase in expression of a network across the two conditions by the majority of subjects provides evidence of the neural response to the increasing demand from $\mathrm{L}$ to $\mathrm{T}$ condition, and therefore links the derived network more unequivocally to the experimental design. The number of subjects violating the rule of increasing expression from $\mathrm{L}$ to $\mathrm{T}$ can be used a statistic to test the null hypothesis and to check for the presence of an ordinal trend (Habeck et al., 2002). The analysis was performed separately on data from the study and test conditions.

Once a covariance pattern was identified that systematically increased in expression from low to titrated demand, we examined the correlation between individual subjects' change in network expression across the two conditions and their scores on the CR proxy measures.

Covariance patterns assign different regional weights to all regional resolution elements (RESELs) included in the analysis, depending on the salience of their covariance contribution. Positive weights (or saliences) imply increasing activation with increasing subject expression of a covariance pattern, while negative voxel weights imply simultaneous decreasing activation with increasing subject expression. This explains how greater subject expression of a covariance pattern accounts for both simultaneous increased and decreased activation at locations of oppositely signed saliences.

Whether its regional weight is reliable (=reliably different from zero) is assessed by a bootstrap estimation procedure (Efron and Tibshirani, 1994) for every voxel. This involved sampling from the subject pool with replacement and repeating the analysis steps that yielded the point estimate results on the resampled data many times $(>500$ iterations). This procedure provides an estimate of the variability of the regional weights in the topography. Smaller variability about its point estimate value indicates that a regional weight is more stable, i.e., its contribution to the covariance pattern can be better generalized to the population from which the subject sample was drawn.

As an indication of a stable regional contribution to the covariance pattern, we required the ratio of the point estimate $w$ to the standard deviation of the resampled estimates $S_{w}$, the inverse coefficient of variation (ICV), to fulfill the relationship

$$
\left|\frac{w}{s_{w}}\right|>1.64
$$

Interpreting the ratio of point estimate to its standard deviation as a normally distributed variable means the above equations fulfils the one-tailed requirement of $P<0.05$. This should be interpreted as a descriptive estimation of 
Table 1

Positive and negative weights according to bootstrap criterion in the topography obtained during the study phase (one-tailed $P<0.05$ )

\begin{tabular}{|c|c|c|c|c|}
\hline$X$ & $Y$ & $Z$ & Level 3 & Level 4 \\
\hline \multicolumn{5}{|l|}{ Positive weights } \\
\hline$x^{2}$ & -67 & -20 & Declive & $*$ \\
\hline 23 & -63 & -24 & Culmen & $*$ \\
\hline 25 & -54 & -22 & Culmen & $*$ \\
\hline \multicolumn{5}{|l|}{ Negative weights } \\
\hline 63 & -43 & -1 & Middle temporal gyrus & $*$ \\
\hline 32 & 21 & -6 & Inferior frontal gyrus & $*$ \\
\hline-2 & -48 & 50 & Precuneus & Brodmann area 7 \\
\hline 6 & -10 & 0 & Thalamus & $*$ \\
\hline-4 & -7 & 6 & Thalamus & $*$ \\
\hline 2 & 2 & 39 & Cingulate gyrus & $*$ \\
\hline 42 & 16 & 1 & Insula & Brodmann area 13 \\
\hline 0 & 7 & $29 *$ & $*$ & \\
\hline 6 & -11 & 13 & Thalamus & Medial dorsal nucleus \\
\hline-30 & 19 & -4 & Inferior frontal gyrus & Brodmann area 47 \\
\hline
\end{tabular}

reliability, rather than a formal test of significance, which would have to proceed in a two-tailed manner.

Once a topography is identified in the analysis of one data set, it can be applied prospectively to any other data set, even if that data set was not part of the original analysis itself. The topography identified in the study phase was applied to the data of the test phase to see whether its expression also correlated with $\mathrm{CR}$ variables.

\section{Results}

\section{Study phase}

OrT CVA was performed on the combined data from study conditions $\mathrm{L}$ and $\mathrm{T}$. The first six principal components served as predictors in a discriminant analysis that probed for a linear combination network whose expression differed maximally on the mean between the $\mathrm{L}$ and $\mathrm{T}$ condition. Although this multiple linear regression model was not significant itself $(P=0.20)$, it yielded the lowest number of subject exceptions (two exceptions) to the rule of increasing expression from $\mathrm{L}$ to $\mathrm{T}$, and the lowest value of the Akaike information criterion. Change in expression of the resulting topography from $\mathrm{L}$ to $\mathrm{T}$ also correlated significantly with both the CR proxy variable NARTIQ $\left(R^{2}=0.24, P<0.05\right)$ as well as performance variables SLS and reaction time difference between $\mathrm{T}$ and $\mathrm{L}$ at test $\left(R^{2}=0.25, P<0.05\right.$ and $R^{2}=0.25, P<0.05$, respectively), providing additional confirmation of having isolated a meaningful neural correlate of task performance.

Fig. 2A-D shows the increase in expression from $\mathrm{L}$ to $\mathrm{T}$ condition and its relationship with functional variables. Fifteen of 17 subjects increased their expression of the covariance pattern in the transition from $\mathrm{L}$ to $\mathrm{T}$ condition (Fig. 2A). The larger the increases of T-L in expression of the covariance pattern, the lower the NARTIQ measures (Fig.
2B), list lengths (SLS) (Fig. 2C) at study, as well as reaction time increases from the $\mathrm{L}$ to the $\mathrm{T}$ test (Fig. 2D). We checked whether NARTIQ was correlated with any of the performance measures, but only found nonsignificant correlations: $R^{2}$ (NARTIQ,SLS) $=0.023 ; R^{2}$ (NARTIQ, RT $\mathrm{T}-\mathrm{L})=0.010$. NARTIQ was the only CR measure that correlated with the T-L difference in pattern expression (correlation with age-scaled vocabulary score: $R^{2}=0.1096$; correlation with years of education: $R^{2}=0.0001$ ).

Stability of the regional weights in the topography was assessed according to the bootstrap estimation procedure outlined above.

Fig. 3 (and Table 1) shows positive and negative regional weights that fulfil the requirement of stability in the bootstrap estimation procedure. Positive weights, whose associated activation increases with increasing subject expression of the covariance pattern and which therefore increase in activation from $\mathrm{L}$ to $\mathrm{T}$ condition for the majority of the subjects, were found in cerebellar locations. Negative weights (associated with decreased activation in $\mathrm{T}$ compared to L) were obtained in Brodmann area 7 (precuneus), anterior cingulate gyrus, bilateral thalamus, right insula, right middle temporal gyrus, and bilateral inferior frontal gyrus.

In summary, most subjects increased the expression of a covariance pattern from $\mathrm{L}$ to $\mathrm{T}$ condition. This increase was, however, not beneficial for performance; to the contrary, less increase in the topography's increase in expression correlated with better performance. Furthermore, subjects with higher NARTIQ showed less increase in the expression of this topography across the two conditions.

Although the topography obtained during the study phase exhibited simultaneous good correlation with both reserve and performance variables, we can remove the influence of RT and SLS from the expression differences of the pattern to probe for a "pure" association with CR. (SLS and RT are already uncorrelated with NARTIQ, and there- 
Table 2

Positive and negative weights according to bootstrap criterion in PC2 during test phase (one-tailed $P<0.05$ )

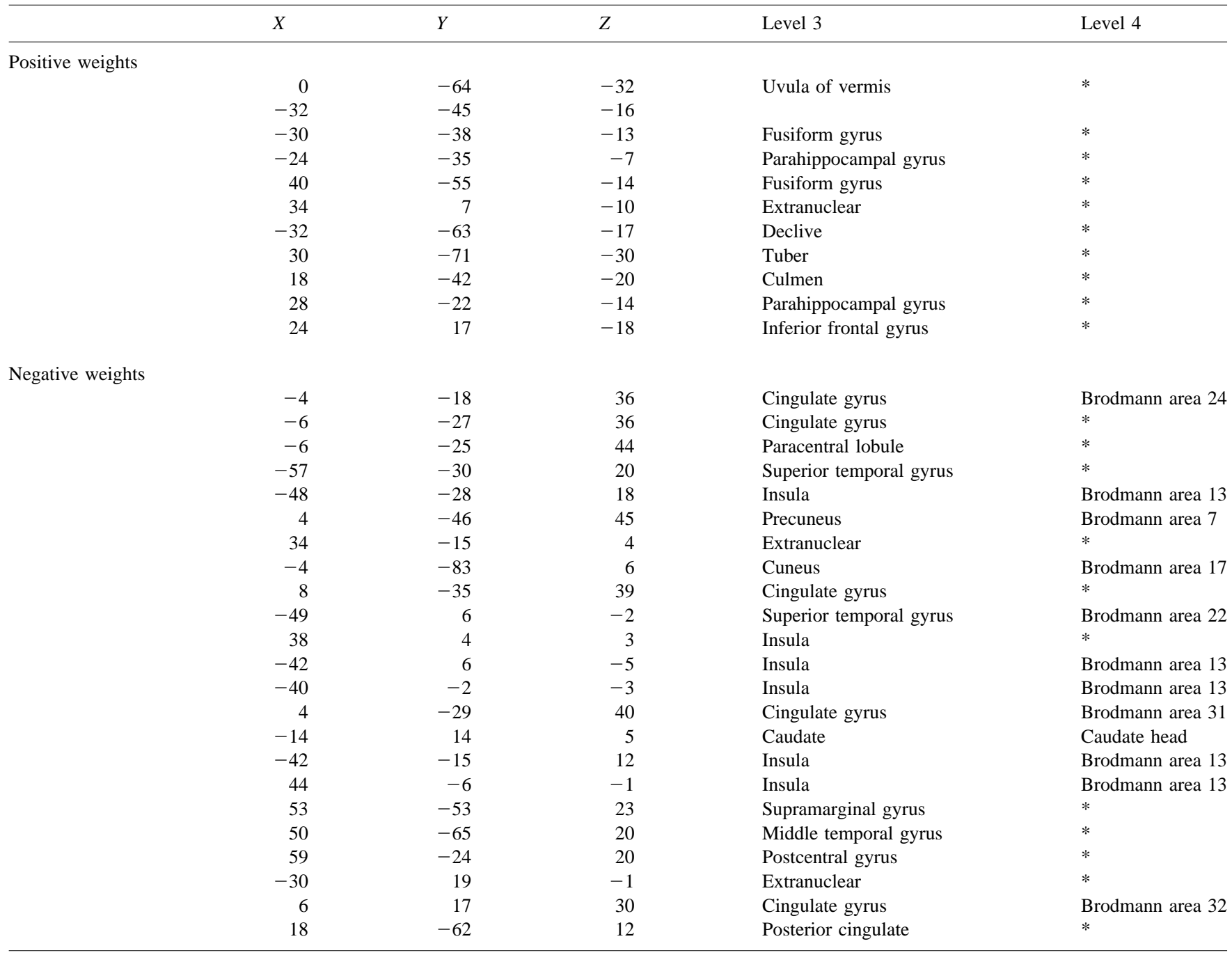

fore need not be removed from it.) The residuals still exhibit a significant correlation with NARTIQ $\left(R^{2}=0.22, P<\right.$ $0.05)$ and the sign of the correlation remained the same (Fig. 4).

\section{Test phase}

Again, OrT/CVA was used on the combined data from $\mathrm{L}$ and $\mathrm{T}$ conditions. Performance and neuropsychological variables also had meaningful associations with brain activation during the test phase, although they could not be integrated into one parsimonious covariance pattern as in the study phase. The difference in the second principal component's expression between $\mathrm{T}$ and $\mathrm{L}$ condition correlated negatively with the reaction time difference between $\mathrm{T}$ and $\mathrm{L}$ condition $\left(R^{2}=0.24, P<0.05\right.$, Fig. 5A). (See also Table 2.) The third principal component's difference in expression correlated with the age-scaled vocabulary score $\left(R^{2}=0.32, P<0.05\right.$, Fig. 5D) and yielded a marginal correlation with the NARTIQ $\left(R^{2}=0.22, P=0.06\right.$, Fig. $5 \mathrm{~B})$, while the fourth principal component's difference in expression correlated with SLS in the T condition $\left(R^{2}=\right.$ $0.36, P<0.01$, Fig. 5C). None of these principal components showed a statistically significant ordinal trend in their subject-task expression as in the study phase.

This dissociation of networks associated with reaction time difference T-L, CR variables (NARTIQ, VocabAgeSc), and SLS is accompanied by a lack of significant regional weights in the bootstrap estimation procedure for principal components 3 and 4. Thus, although we could establish significant global associations between NARTIQ and SLS on the one hand and brain activation on the other, these associations could not be localized to specific RESELs with a one-tailed $P$-level $P<0.1$. The associations remained strictly between pattern expression and behavioral variables.

We could establish robust regional contributions to the second principal component $(|\mathrm{ICV}|>1.64)$. Therefore, the 


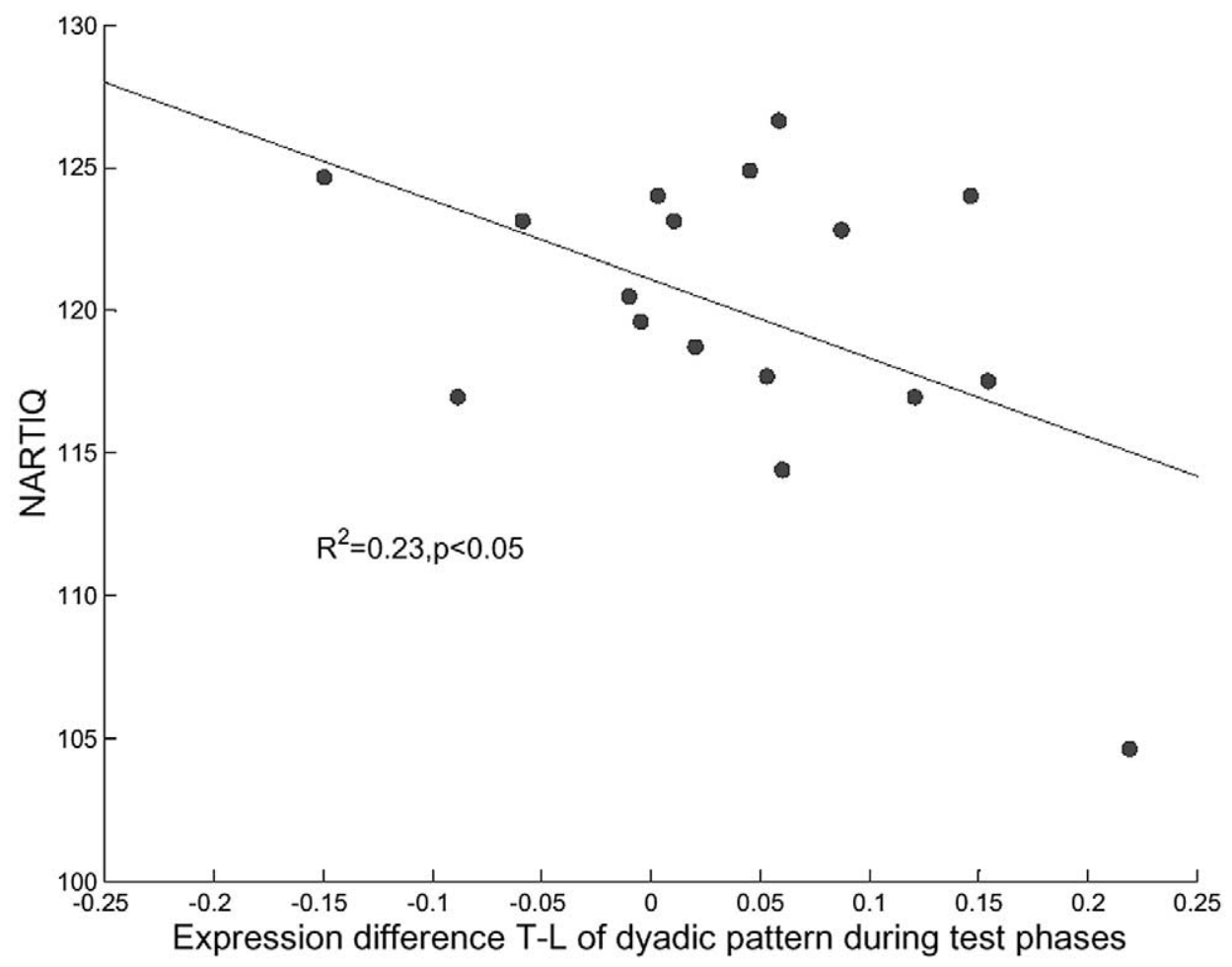

Fig. 7. Correlation between NARTIQ and the expression difference T-L obtained by applying the topography found during the study phase prospectively to the test phase. When SLS and reaction time difference RT T-L were partialed out, the correlation worsened slightly to $R^{2}=0.21, P=0.06$ (graph not shown).

reaction time increase from $\mathrm{L}$ to $\mathrm{T}$ condition was associated with a significant portion of the variance in the neural data, while also permitting robust inference of the regional activation pattern.

Anatomical loci of principal component 2 whose regional weights fulfil the robustness requirement for the bootstrap resampling procedure included those of the covariance pattern found during the study phase (see Fig. 6). Changes of activation at loci that had stable regional weights for both topographies showed the same directionality in their correlation with the reaction time increase from $\mathrm{L}$ to $\mathrm{T}$ during test.

\section{Connections between study and test phase}

We prospectively applied the topography derived in the study phase to data from the test phase. The difference in expression T-L in the test phase correlated significantly with NARTIQ (Fig. 7) and also displayed an ordinal trend (four subject exceptions, $P<0.05$ ).

Thus, despite the difficulty in identifying a single network in the data collected during the test phase, the expression difference T-L of the pattern obtained during the study phase was also a measure of CR for these data. The correlation worsened slightly when SLS and RT T-L were partialed out from the expression difference T-L: $R^{2}=0.21, P$ $=0.06$. Furthermore, we also obtained a significant correlation between the expression difference T-L of the topog- raphy during study and the prospective application during test $\left(R^{2}=0.43, P<0.01\right)$. These findings indicate generalizability of the study topography beyond the data from the study phase of this experiment.

\section{Discussion}

Activation patterns were recovered from the study and test phases using regional covariance methods. In each activation pattern, the change in pattern expression with increased cognitive load correlated with subject memory performance and $\mathrm{CR}$ variables.

The study phase revealed an activation pattern that exhibited an ordinal trend, i.e., increasing subject expression for 15 of 17 subjects from $\mathrm{L}$ to $\mathrm{T}$ condition, and was associated with CR, i.e., showing greater changes in expression for individuals with lower CR. This activation pattern offers a parsimonious account for both performance and CR variables: change in network expression across the $\mathrm{L}$ and $\mathrm{T}$ conditions correlated significantly with RT, SLS, and NARTIQ. Subjects who activated this network the most in their transition from $\mathrm{L}$ to $\mathrm{T}$ condition were capable of working only with the lowest SLS and had the lowest levels of the CR (lower NARTIQ). In addition, they showed the least increase from $\mathrm{L}$ to $\mathrm{T}$ in reaction time at test, possibly because they have to search through a smaller array of successfully remembered shape items. These results are 
congruent with those of a GLM univariate analysis (Stern et al., 2003) which established significant correlation of the T-L activation maps with the reserve variable IQ in several locations during both study and test. In the computation of these brain maps, SLS and reaction time difference T-L were partialed out from the T-L difference data in order to isolate a pure association between CR (IQ) and brain activation. For the same reasons, we removed the influence of RT and SLS from the expression differences of the study topography. The residuals still exhibited a significant correlation with NARTIQ. Thus, network expression is related to $\mathrm{CR}$ in a manner that is independent of actual task performance.

In the analysis of the test phase, no single activation pattern was associated with task performance and CR. In particular, no ordinal trend could be detected as in the study phase. Instead, three separate principal components were noted that related to $\mathrm{CR}$ and performance in different ways. The second principle component contained some of the same brain areas that were noted in the network isolated during the study phase. Increased expression of this network from $\mathrm{L}$ to $\mathrm{T}$ correlated with decreased reaction time differences. Furthermore, the network also included the parahippcompal and lingual gyri, whose increase in activation from $\mathrm{L}$ to $\mathrm{T}$ correlated negatively with the reaction time increase.

Change in expression from $\mathrm{L}$ to $\mathrm{T}$ in principal component 3 correlated with age-scaled vocabulary scores and marginally with NARTIQ, both indices of CR. Change in expression from $\mathrm{L}$ to $\mathrm{T}$ in principal component 4 correlated with SLS. Principal components 3 and 4 did not present any robust regional weights that fulfilled the threshold requirement indicated by a bootstrap estimation procedure. Thus, while there were significant associations between specific measures and brain activation as expressed by the entire covariance pattern, the corresponding neural correlates could not be sufficiently localized in the bootstrap estimation procedure and the activation patterns remained nonfocal.

The brain regions showing decreased expression in the network identified during study included Brodmann area 7 (precuneus), anterior cingulate gyrus, bilateral thalamus, right insula, right middle temporal gyrus, and bilateral inferior frontal gyrus. Some of these regions were also noted in the second principal component isolated during the test phase. These brain regions are suggestive of a frontoparietal network previously found to underlie both overt and covert shifts of spatial attention (Beauchamp et al., 2001; Burgess et al., 2001; Calhoun et al., 2001). This allows the following interpretation of the network: the event-related parametric maps that are obtained in the within-subject GLM estimation average over all item presentations in the $\mathrm{T}$ condition. It may have been that in the titrated condition, not all subjects were able to encode serially presented items with sustained attention throughout the study phase. This loss of attention would be reflected in the deactivation of the spatial attention network for 15 out of 17 subjects from $\mathrm{L}$ to $\mathrm{T}$ condition. Consistent with this interpretation, subjects who were least susceptible to fatigue were the ones with higher CR and superior performance, i.e., with higher NARTIQ and longer list lengths.

Forward application of a covariance pattern to new data sets that did not enter into the original analysis is one of the attractive features of multivariate analyses. When we prospectively applied the topography obtained during the study phase to the functional data from the test phase, we found that expression of this topography during test correlated significantly with NARTIQ. Thus, differential activation of the study topography as a function of CR was comparable in both the study and test phase, despite both having quite different covariance structures, indicating different putative cognitive mechanisms during study and test. From the successful forward-application of the study topography to the test phase, we might speculate that the network found in both the study and test phase generalizes to other nonverbal recognition tasks in general, and may, in part, underlie the different approach to tasks used by individuals with more and less CR. This idea lies at the heart of the CR hypothesis and will be tested in future experiments.

\section{References}

Alexander, G.E., Mentis, M.J., Van Horn, J.D., Grady, C.L., Berman, K.F., Furey, M.L., Pietrini, P., Rapoport, S.I., Schapiro, M.B., Moeller, J.R., 1999. Individual differences in PET activation of object perception and attention systems predict face matching accuracy. Neuroreport 10 (9), 1965-1971.

Beauchamp, M.S., Petit, L., Ellmore, T.M., Ingeholm, J., Haxby, J.V., 2001. A parametric fMRI study of overt and covert shifts of visuospatial attention. Neuroimage 14 (2), 310-321.

Burgess, N., Maguire, E.A., Spiers, H.J., O’Keefe, J., 2001. A temporoparietal and prefrontal network for retrieving the spatial context of lifelike events. Neuroimage 14 (2), 439-453.

Burnham, K.P., Anderson, D.R., 2002. Model Selection and Multimodel Inference. Springer Verlag, New York.

Cabeza, R., Grady, C.L., Nyberg, L., McIntosh, A.R., Tulving, E., Kapur, S., Jennings, J.M., Houle, S., Craik, F.I., 1997a. Age-related differences in neural activity during memory encoding and retrieval: a positron emission tomography study. J. Neurosci. 17 (1), 391-400.

Cabeza, R., McIntosh, A.R., Tulving, E., Nyberg, L., Grady, C.L., 1997b. Age-related differences in effective neural connectivity during encoding and recall. Neuroreport 8 (16), 3479-3483.

Cabeza, R., Anderson, N.D., Locantore, J.K., McIntosh, A.R., 2002. Aging gracefully: compensatory brain activity in high-performing older adults. Neuroimage 17 (3), 1394-1402.

Calhoun, V.D., Adali, T., McGinty, V.B., Pekar, J.J., Watson, T.D., Pearlson, G.D., 2001. fMRI activation in a visual-perception task: network of areas detected using the general linear model and independent components analysis. Neuroimage 14 (5), 1080-1088.

Efron, B., Tibshirani, R.J., 1994. An Introduction to the Bootstrap. CRC Press, LLC, New York.

Elias, M.F., Beiser, A., Wolf, P.A., Au, R., White, R.F., D’Agostino, R.B., 2000. The preclinical phase of Alzheimer disease: a 22-year prospective study of the Framingham Cohort. Arch. Neurol. 57 (6), 808-813.

Grady, C.L., McIntosh, A.R., Rajah, M.N., Beig, S., Craik, F.I., 1999. The effects of age on the neural correlates of episodic encoding. Cereb. Cortex 9 (8), 805-814. 
Habeck, C. G., Stern, Y., Posner, H. B., Moeller, J. R., 2002. Regional covariance analysis of event-related fMRI. SFN Abstracts.

McIntosh, A.R., Bookstein, F.L., Haxby, J.V., Grady, C.L., 1996. Spatial pattern analysis of functional brain images using partial least squares. Neuroimage 3 (3 Pt 1), 143-157.

Qiu, C., Backman, L., Winblad, B., Aguero-Torres, H., Fratiglioni, L., 2001. The influence of education on clinically diagnosed dementia incidence and mortality data from the Kungsholmen Project. Arch. Neurol. 58 (12), 2034-2039.

Reuter-Lorenz, P., 2002. New visions of the aging mind and brain. Trends Cogn. Sci. 6 (9), 394.

Scarmeas, N., Levy, G., Tang, M.X., Manly, J., Stern, Y., 2001. Influence of leisure activity on the incidence of Alzheimer's disease. Neurology 57 (12), 2236-2242.

Scarmeas, N., Zarahn, E., Anderson, K. E., Hilton, J., Flynn, J., Van Heertum, R.L., Sackeim, H.A., Stern, Y., 2003. Cognitive reserve modulates functional brain responses during memory tasks: a PET study in healthy young and elderly subjects. Neuroimage 19, 12151227.

Schmand, B., Smit, J.H., Geerlings, M.I., Lindeboom, J., 1997. The effects of intelligence and education on the development of dementia. A test of the brain reserve hypothesis. Psychol. Med. 27 (6), 1337-1344.

Stern, Y., 2002. What is cognitive reserve? Theory and research application of the reserve concept. J. Int. Neuropsychol. Soc. 8 (3), $448-460$

Stern, Y., Moeller, J.R., Anderson, K.E., Luber, B., Zubin, N.R., DiMauro, A.A., Park, A., Campbell, C.E., Marder, K., Bell, K., Van Heertum, R., Sackeim, H.A., 2000. Different brain networks mediate task performance in normal aging and AD: defining compensation. Neurology 55 (9), 1291-1297.

Stern, Y., Zarahn, E., Hilton, H.J., Flynn, J., DeLaPaz, R., Rakitin, B., 2003. Exploring the neural basis of cognitive reserve. J. Clin. Exp. Neuropsychol. 25, 691-701.

Worsley, K.J., Poline, J.B., Friston, K.J., Evans, A.C., 1997. Characterizing the response of PET and fMRI data using multivariate linear models. Neuroimage 6 (4), 305-319.

Zarahn, E., 2000. Testing for neural responses during temporal components of trials with BOLD fMRI. Neuroimage 11 (6 Pt 1), 783-796.

Zarahn, E., 2002. Using larger dimensional signal subspaces to increase sensitivity in fMRI time series analyses. Hum. Brain Mapp. 17 (1), 13-16. 